\section{Rate of simple motor responding as a function of coaction, sex of the participants, and the presence or absence of the experimenter*}

\author{
D. W. CARMENT and MARGARET LATCHFORD \\ McMaster University, Hamilion, Ontario, Canada
}

Male and female Ss performed a simple motor task, half alone and half coacting. Half of these $S$ s responded while $E$ was not present. The dependent variable was rate of responding. Ss responded more rapidly and social facilitation appeared only when $\mathbf{E}$ was present. In E's absence rate of responding declined and solitary Ss responded more rapidly than coacting Ss. Implications for a learned-drive interpretation are noted.

There is some evidence that indicates that coaction effects occur only when the coactors perceive that their performance is under surveillance and is being evaluated. Cottrell (1968), for example, was unable to find any facilitation of performance in the presence of a disinterested audience. This suggests, as well, that the Ss were unaffected by potential evaluation on the part of $E$ who the $S s$ were aware was in an adjacent control room. In fact, few, if any, of the experiments concerned with social facilitation have paid attention to the role of $E$. Yet, if anticipation of positive or negative outcomes is a factor of importance, Es involvement would appear to be crucial.

The experiment reported here was designed to assess the effects of the presence or absence of $E$ on the rate of responding of male and female coacting and solitary Ss.

\section{SUBJECTS AND DESIGN}

There were $96 \mathrm{Ss}, 48$ males and 48 femaies, chosen at random from an introductory psychology course. Half of these Ss performed alone and half with a coactor of the same sex. These sets were further subdivided so that in half the cases E was present during the experimental session and in the remainder $E$ was absent. This results in eight experimental conditions with 12 Ss in each condition.

APPARATUS AND PROCEDURE

The apparatus consisted of panel-mounted self-centering toggle switches connected to programming and timing units behind a partition in the same room.

The Ss were brought to the room, seated comfortably before a panel, and those in the solitary conditions then were instructed: "This experiment is concerned with some aspects of coordination. We

\footnotetext{
* This research was supported by Grant No. PA 0332 from The National Research Comeit of Canado and a Department of Labour.
}

would like you to move this lever back and forth something like this... with your thumb and first two fingers clasped around it. Please use only one hand throughout the experiment. This experiment lasts for $10 \mathrm{~min}$, after 5,1 will stop you and you may rest for $3 \mathrm{~min}$. Any questions? ... Fine. you may start when I say go."

The $\mathrm{E}$ then went behind the panel, turned on the apparatus, and gave the signal to begin.

In the coacting conditions the following instructions were added: "One last thing, there is no connection between the two panels. What one of you does, doesn't affect the other. We are simply running two people at a time to get through the experiment faster. Please do not talk to each other while you are working."

In the E-absent conditions the following instructions were included: "I have some work to do across the hall, so I will not be in the room while you are responding. I will return in $5 \mathrm{~min}$, at which time you may have a 3-min rest." After saying "go," the E left the room.

All Ss responded for $10 \mathrm{~min}$ in two

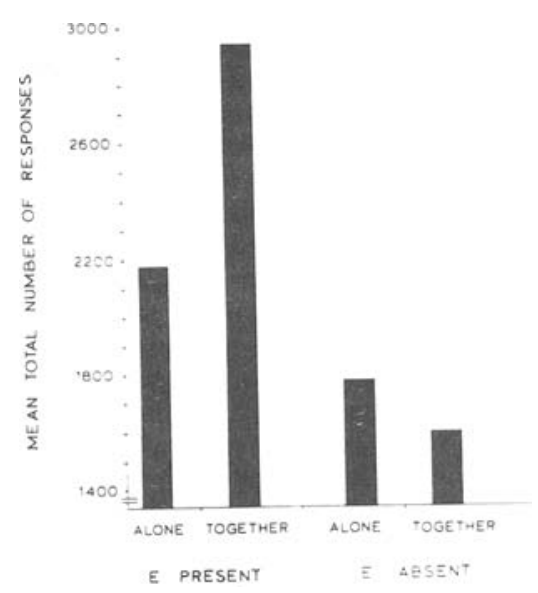

Fig. 1. Mean total number of responses as a function of presence or absence of $\mathbf{E}$ and coaction. 5-min segments with an intervening 3-min rest interval. Ss were not allowed to talk about the experiment during this interval. It should be noted that under all conditions $E$ was a female.

\section{RESULTS}

The analysis of variance consisted of three between factors (coaction, sex, and the presence or absence of E) and two within factors (time and segments). The analysis revealed a significant main effect of E's presence or absence ( $F=22.72$, $\mathrm{df}=1 / 88, p<.001)$. Ss in the E-absent condition made fewer responses than those in the E-present condition. However, this finding must be modified in view of a significant $\mathrm{E}$ by Coaction interaction $(\mathrm{F}=3.97, \mathrm{df}=1 / 88, \mathrm{p}<.05)$ which is illustrated in Fig. 1. It can be seen, and this is supported by Scheffe multiple comparisons, that coacting $S s$ with $E$ present respond faster than all other Ss. Solitary Ss with E present respond more rapidly than $S$ s in both $E$-absent groups. In the E-absent groups solitary Ss make more responses than coacting Ss. There were no differences attributable to the sex of the Ss.

Within Ss there is a significant overall increase in responding over time $(F=29.61, \quad d f=4 / 352, p<.001)$ and $a$ Time by $\mathrm{E}$ interaction $(\mathrm{F}=2.99$, $\mathrm{df}=4 / 352, \mathrm{p}<.05$ ) since the rate of increase in responding is greater for all $\mathrm{Ss}$ in the presence of $E$ than is the case for those performing with $E$ absent.

There also is a significant segment effect $(F=56.23, \mathrm{df}=1 / 88, \mathrm{p}<.001)$ based on the greater overall rate of responding during the second segment as compared with the first segment.

\section{DISCUSSION}

It is clear that the presence of $E$ has an energizing effect on the performance of both solitary and coacting Ss. Under this condition the usual coaction effects also are evident. However, not only does the rate of responding decline when $E$ is absent, but the coaction effects disappear as we]l. This offers rather strong support for Cottrell's (1968) view that anticipated evaluation is necessary for performance to be facilitated. Apparently, under the conditions of this experiment, a coactor is not perceived as an evaluator. This could be because no information was made available to the $S$ s as to how well they were performing. This possibility may be clarified by experiments underway which include a "knowledge of results" with $\mathrm{E}$ absent condition. A generalized arousal theory of social facilitation would have some difficulty with the present data, whereas it fits readily into a learned drive 
framework.

The analysis of performance over time offered little of interest other than the information that differences appeared early and remained throughout the experimental session.
REFERENCE

COTTRELL, N. B. Performance in the presence of other human beings. Mere presence, audience, and affiliation effects. In $\mathrm{E}$. C. Simmel, R. A. Hoppe, and G. A. Milton (Lis.) Social facilitation and imitative behavior. Boston: Allyn \& Bacon, 1968. Pp. 91-110.

\section{Words functioning simultaneously as negative operant reinforcers and unconditioned stimuli}

\author{
ANDREW T. ABELL* \\ Southeastern Louisiana College, Hammond, La. 70401
}

To continue an analysis of earlier findings, data were secured from 112 undergraduates in a study employing an identical Ss design. A conditioning device made of paper (reinforcer form) allowed exposures of unpleasant words and of neutral ones to be dependent upon motor responses to two CVCs. As predicted, Ss tended to expose neutral words rather than unpleasant ones, and they subsequently rated the $\mathrm{CVC}$ associated with unpleasant words as less pleasant than the other trigram.

This study continues an analysis (Abell, 1970) of earlier results (Abell, 1969a, b, c). Abell (1970) recently demonstrated the contemporaneous operation of pleasant words as UCSs and positive operant reinforcers, thereby revealing one source of earlier findings (Abell, 1969a, b, c). The present study was designed to determine whether or not unpleasant words can function simultaneously as UCSs and suppressors of operant behavior. Confirmation would indicate that words functioning simultaneously as negative operant reinforcers (via punishment) and as UCSs also contributed to the earlier results (Abell, 1969a, b, c).

The hypotheses were the following: (1) The tendency to respond to CVCs followed by unpleasant words is less than that to CVCs followed by neutral words; (2) CVCs preceding unpleasant words are rated as less pleasant than those preceding neutral words.

\section{SUBJECTS}

Students in seven introductory psychology classes (56 males and 56 females) served as Ss during regular class periods. They were given no advance information about the experiment.

$$
\text { MATERIALS }
$$

Two CVCs, XIB and ZUJ, which have the same scaled meaningfulness (Noble, 1961), and eight evaluative words, were

*Beginning September 1970, at Westminster College, New Wilmington, Pa. 16142. presented in an identical Ss design by means of reinforcer forms like those used earlier (Abell, 1970). The evaluative words, four neutral and four unpleasant, were chosen from previous ratings on a 7-point scale (Abell, 1970, Table A). The neutral words were the same four used before (Abell, 1970). The unpleasant words, together with their medians $(1.000$ represents the extreme of unpleasantness), are the following: agony, 1.245; vomit, 1.245; terror, 1.391; and failure, 1.411. As in earlier studies (Abell, 1969a, b, c, 1970) a rating sheet was included with each reinforcer form.

As before (e.g., Abell, 1970), the sets of materiais incorporated various controls. In half the forms, XIB concealed an unpleasant word and ZUJ, a neutral one (Tab Placement $\mathrm{U}$ ); in the others, the reverse was true (Tab Placement N). These different tab placements, together with two different arrangements of words on undersheets (A and B) and two slightly different rating sheets ( 1 and 2), produced eight different booklets: U-A-1, U-A-2, U-B-1, U-B-2, N-A-1, N-A-2, N-B-1, and N-B-2.

\section{PROCEDURE}

As the design required a multiple of eight Ss per class, from four to seven students were eliminated in a random way from each class. Then, the eight different kinds of booklets, which had been stacked earlier in a sequence randomized within each block of eight, were distributed by rows to the remaining students. Thus, a total of $14 \mathrm{Ss}$ was assigned to each of the eight control conditions.

As in earlier studies (Abell, 1969a, 1970), Ss were instructed to use a recorded sequence of words ("open," "choose," and "close") to pace responses to the forms; they were allowed about a minute's rest between pages; and they subsequently rated the CVCs for pleasantness on a 7-point scale.

RESULTS AND DISCUSSION

Neutral words were exposed more often than unpleasant ones by $71 \mathrm{Ss}$ and less frequently, by 34 ; the two types were exposed equally often by 7 . The sign test for large samples (Siegel, 1956) produced a $\mathrm{z}$ of 3.32 (one-tailed $\mathrm{p}<.0005$ ). The CVC associated with unpleasant words was judged, subsequently, as less pleasant than the other one by $63 \mathrm{Ss}$ and as more pleasant, by 29 ; the two were evaluated the same by 20 . The sign test yielded a $z$ of 3.44 (one-tailed $\mathrm{p}<.0003$ ).

The confirmation of both hypotheses demonstrates that words can function simultaneously as - negative operant reinforcers and UCSs. This demonstration, together with the one that showed that words can function concurrently as positive operant reinforcers and UCSs (Abell, 1970), helps explain earlier results (Abell, 1969a, b, c). Apparently, both positive reinforcement and punishment operated to produce the difference in strength of operant response to trigrams that was found in the initial (Abell, $1969 \mathrm{~b}$ ), as well as in each confirming (Abell, 1969a, c), gross-comparison study. Similarly, the difference in evaluative meaning of trigrams that was found in those early studies (Abell, 1969a, b, c) seems to reflect the operation of two processes: (1) the conditioning of pleasantness to one of the trigrams; (2) the conditioning of unpleasantness to the other.

\section{REFERENCES}

ABELL, A. T. A test of reinforcer forms with flaps. Perceptual \& Motor Skills, 1969a, 29, 984-986.

ABELL, A. T. Words functioning simultaneously as operant and respondent reinforcers: Preliminary study. Psychological Reports, 1969 b, 24, 123-133.

ABELL, A. T. Words functioning simultaneously as operant and respondent reinforcers: Replication. Psychological Reports, 1969c, 25, 975.

ABELL, A. T. Words functioning simultaneously as positive operant reinforcers and unconditioned stimuli. Psychonomic Science, $1970,19,325-326$.

NOBLE, C. E. Measurements of association value (a), rated associations (a'), and scaled meaningfulness $\left(\mathrm{m}^{\prime}\right)$ for the 2100 CVC umbinations of the English alphabet. Psychological Reports, 1961, 8, 487-521.

SIEGEL, S. Nonparametric statistics for the behavioral sciences. New York: McGraw-Hill, 1956. 